\title{
Scan-noise and Drift Correction in the STEM
}

\section{Lewys Jones}

Department of Materials, University of Oxford, 13 Parks Road, Oxford, UK OX52AF

lewys.jones@materials.ox.ac.uk

Modern scanning transmission electron microscopes (STEMs) now routinely achieve atomic resolution, and these instruments are finding homes in more and more institutions worldwide. The "S" in STEM denotes that these microscopes record their images in a scanned (serial) manner, scanning pixel by pixel to build up the data. The benefit of this is that not only can images be obtained at this impressive resolution, but analytical data can also be obtained, such as X-ray or energy-loss spectroscopy signals. Unfortunately, the drawback of scanned acquisition is that it may take up to a few tens of seconds to record an entire frame, and the operator then has to be concerned about the stability of the microscope, the sample stage, and even the room.

In STEM, instabilities at high frequencies will result in so-called scan-noise, and this is clearly seen in the jagged and torn columns of the atomic-resolution dark-field image shown in the left half of Figure 1. This effect arises from the very way the data is recorded: the image signal is desired as a position series, but serial acquisition is in essence a time series. Any deviation between these two appears as scan-noise.

Fortunately, it is possible to observe these characteristic distortions and to mitigate their effects [1]. Using the Jitterbug plug-in for Digital Micrograph from HREM Research [2], the as-recorded image is scrutinized on a pixel-by-pixel basis. The magnitude of the scan distortion at each point is determined by comparing the region about each pixel with neighboring scan lines above and below. With all the distortions diagnosed, it is possible to restore an image with significantly reduced scan-noise (Figure 1, right half).

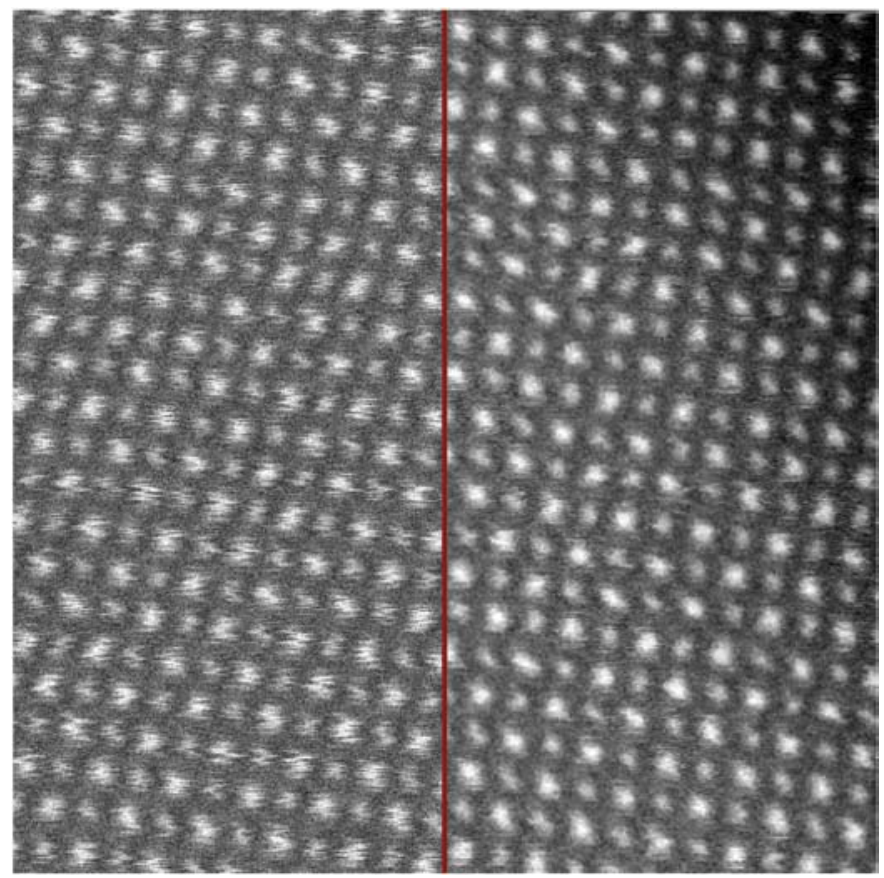

Figure 1: Dark-field STEM image of [100] oriented $\mathrm{SrTiO}_{3}$, before scan-noise and drift correction (left) and after (right). In the left half of the image, the atomic columns appear jagged and torn, and the square symmetry of the crystal lattice is heavily sheared. Image width $=5 \mathrm{~nm}$.
Stage/sample drift can also be a problem during the scan, and this leads to images that appear to be sheared. In this case, angles of the crystal lattice are incorrect, and the image magnification may be distorted. Fortunately, this is also easily diagnosed and corrected by applying an opposing image shear. Looking again at Figure 1, even though the sample is known to have a cubic structure, the lattice planes are far from perpendicular, and the lattice parameter appears different in different directions. Using the Jitterbug plug-in, and specifying the appropriate base vector directions and lengths, this drift also can be corrected. Thus, the lattice angles and lengths can be restored to their correct values.

Another indicator of scan-noise is the presence of artifacts in the Fourier transform (Figure 2). The most severe artifacts here are the long diffuse streaks running vertically from the low-order spots. After the scan-noise correction these vertical streaks are significantly reduced, and the visibility of the spots is improved. If image drift can be corrected, then the positions and angles between spots may be rectified, making diffractogram magnification calibration more accurate. The removal of the Fourier artifacts, along with the improved visibility of the Fourier spots, also suggests that there have been improvements in the signal-to-noise ratio (SNR) and resolution, respectively.

The Jitterbug plug-in also includes tools to quantify the performance of STEM images. Resolution is measured in real-space by the fitting of two-dimensional Gaussians to many atomic columns; SNR is measured using the root-mean-square power of real-space line profiles through many different directions [1].

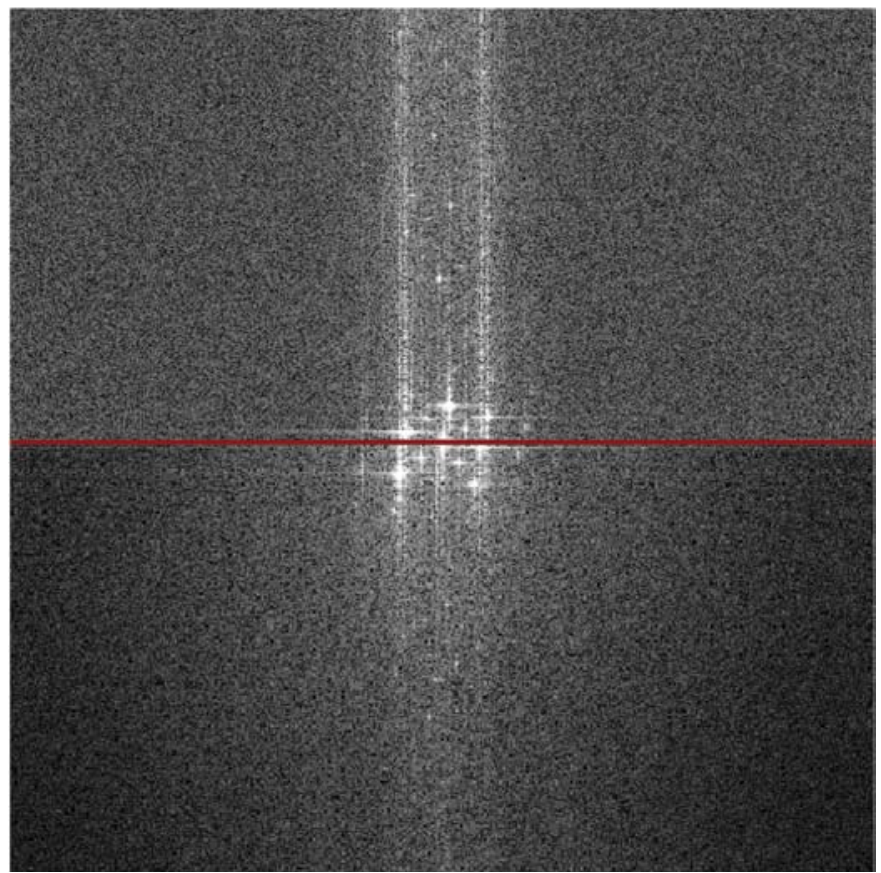

Figure 2: Fourier transforms of the images shown in Figure 1 showing the original FT (top half) and the restored FT (bottom). 


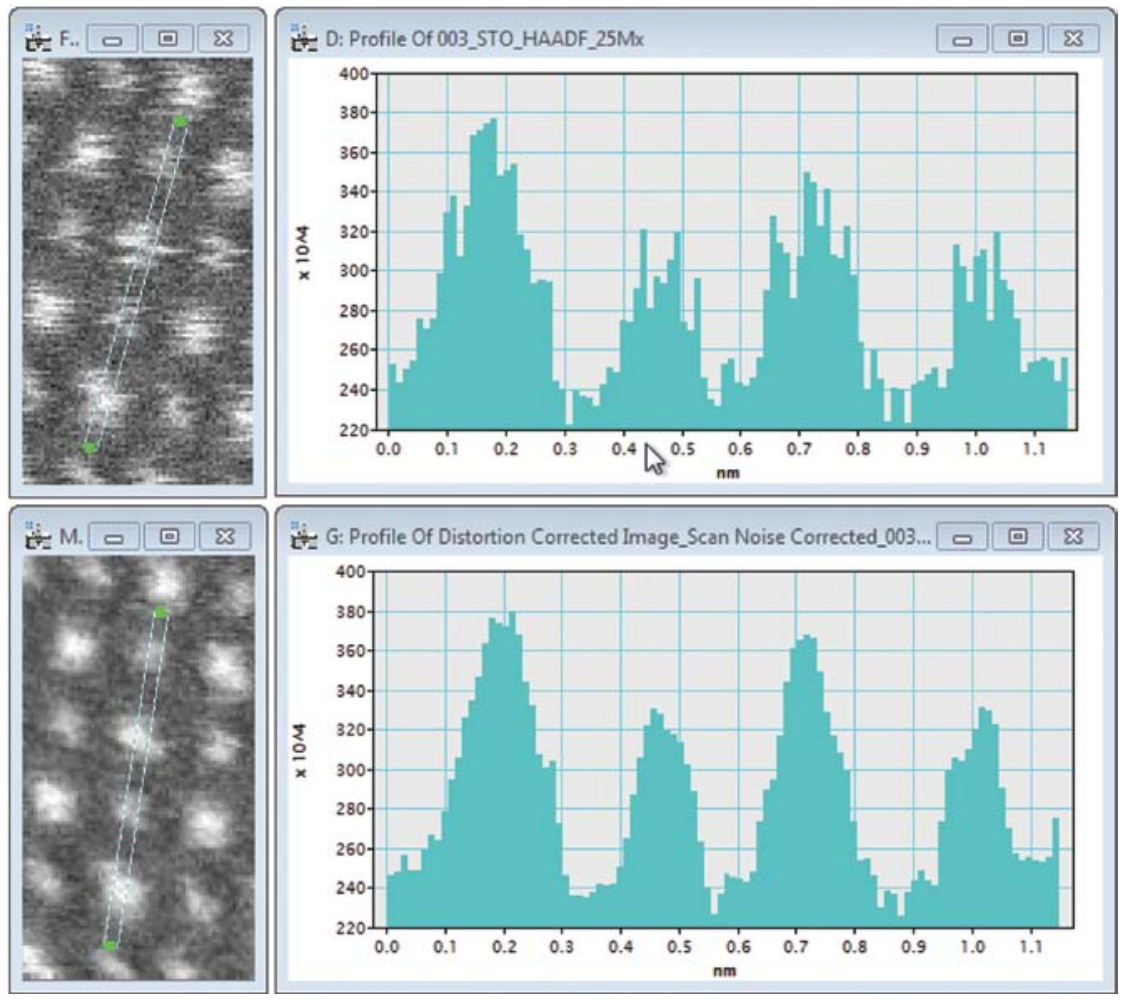

Figure 3: Example line-profiles taken from the same positions near the lower-left corner of the data shown in Figure 1 both before (top) and after (bottom) scan-noise correction.
To demonstrate the improvement in image quality more quantitatively, line profiles through the same points before and after scan-noise and drift compensation are shown in Figure 3.

In this example, image resolution was improved from $88.7 \mathrm{pm}$ to $75.1 \mathrm{pm}(15.3 \%$ better), and SNR was improved from 2.04 to $3.76(84.3 \%)$. Importantly the integrated signal under atomic-column peaks remains the same, making this method suitable for use with quantitative STEM techniques.

Clearly it is not possible to improve resolution and SNR beyond the performance of the microscope. In fact, what the plug-in achieved here was to retrieve the performance the microscope was originally capable of, before corruption by scan-noise and drift. The performance boost offered by this technique makes it easier for users to get the best possible results from their instruments.

\section{References}

[1] L Jones and PD Nellist, Microscopy and Microanalysis 19 (2013) 1050-60

[2] Available from HREM Research Inc.: (www.hremresearch.com). Digital Micrograph is a trademark of Gatan Inc.

\section{Expand your Knowledge of Microscopy with} MSA

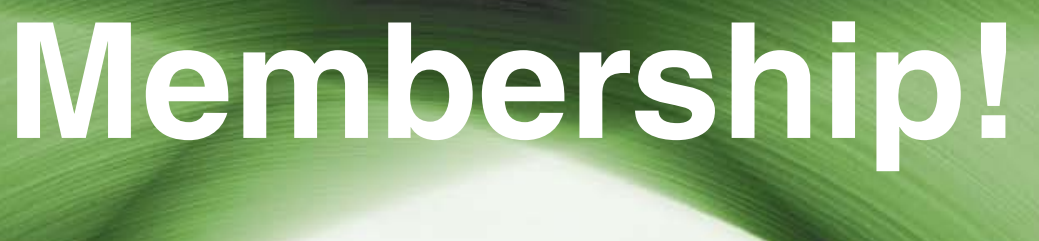

Whether your primary focus is in optical, electron or scanning probe microscopy, the biological or the physical sciences, MSA takes your knowledge to the next level!

\section{Members Receive:}

- A personal subscription to MSA's official journal, Microscopy and Microanalysis, and MSA's popular bi-monthly magazine, Microscopy Today.

- Peer Networking through the Society's Focused Interest Groups and Local Affiliated Societies.

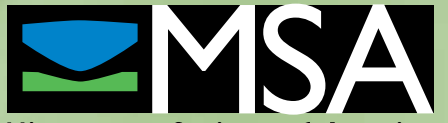

Microscopy Society of America
- Plus discounts on books, journals and other educational materials.

- MSA Awards Programs, Scholarships, Speaker Opportunities, and much more! 